\title{
The desire to hasten death: Using Grounded Theory for a better understanding "When perception of time tends to be a slippery slope"
}

Palliative Medicine

2015, Vol. 29(8) 71I-719

(C) The Author(s) 2015

Reprints and permissions:

sagepub.co.uk/journalsPermissions.nav DOI: $10.1177 / 0269216315577748$ pmi.sagepub.com

@SAGE

\author{
Martina Pestinger', Stephanie Stiel'2,3, Frank Elsner', \\ Guy Widdershoven ${ }^{4}$, Raymond Voltz ${ }^{5}$, Friedemann Nauck ${ }^{6}$ \\ and Lukas Radbruch ${ }^{7,8}$
}

\begin{abstract}
Background: Some patients with advanced and progressive diseases express a desire to hasten death.

Aim: This study evaluated the motivations of patients expressing such a desire in a country with prohibitive legislation on euthanasia and physician-assisted suicide.

Design: A modified form of Grounded Theory was used.

Setting/participants: Patients from the departments of palliative medicine in three hospitals in Germany were recruited when they had made a statement or request to hasten death. Participants were interviewed face to face. Recruitment was stopped with 12 participants because of data saturation.

Results: Thematic analysis revealed three main motivational themes: self-determination, agony, and time. Expectations toward health professionals, attitudes toward death, and secureness related to the end of life were additional main themes emerging from the analysis. Conclusions: The desire to hasten death may be used as an extreme coping strategy to maintain control against anticipated agony. Patients expected health professionals to listen to and respect their experiences. Emerging hypotheses included the following: (a) patients try to balance life time and anticipated agony, and the perception of time is distressing in this balancing act; (b) anticipated images of agony and suffering in the dying process occur frequently and are experienced by patients as intrusive; (c) patients expressing a desire to hasten death are in need of more information about the dying process; and (d) patients wanted their caregivers to listen to and respect their wish to hasten death, and they did not expect the caregivers to understand this as an order to actually hasten their death.
\end{abstract}

\section{Keywords}

Desire to die, physician-assisted suicide, euthanasia, palliative care, anticipated suffering, agony

What is already known about the topic?

- One out of 10 patients with advanced progressive disease considers hastening death, although only a minority of these patients talk about their wish for hastened death with their caregivers.

'Department for Palliative Medicine, RWTH Aachen University, Aachen, Germany

${ }^{2}$ Department of Palliative Medicine, University Hospital Erlangen,

Friedrich-Alexander-Universität Erlangen-Nürnberg, Erlangen, Germany

${ }^{3}$ Comprehensive Cancer Center (CCC) Erlangen-EMN, University

Hospital Erlangen, Friedrich-Alexander-Universität Erlangen-Nürnberg,

Erlangen, Germany

${ }^{4}$ Department of Medical Humanities, EMGO Institute for Health and

Care Research, VU University Medical Center, Amsterdam, The

Netherlands

${ }^{5}$ Department for Palliative Medicine, University Hospital Cologne,

Cologne, Germany
${ }^{6}$ Department for Palliative Medicine, University Hospital Göttingen, Göttingen, Germany

${ }^{7}$ Department of Palliative Medicine, University Hospital Bonn, Bonn, Germany

${ }^{8}$ Centre for Palliative Care, Malteser Hospital Seliger Gerhard Bonn/ Rhein-Sieg, Bonn, Germany

Corresponding author:

Lukas Radbruch, Department of Palliative Medicine, University Hospital Bonn, Sigmund Freud Str. 25, 53127 Bonn, Germany.

Email: Lukas.radbruch@malteser.org 


\section{What this paper adds?}

- The desire to hasten death is an extreme coping strategy for intrusive emotional and cognitive experiences, often related to anticipated images of agony and suffering in the dying process.

- Patients do not perceive their desire to hasten death and the provision of palliative care as mutually exclusive.

- Patients wanted their caregivers to listen to and respect their wish to hasten death, and they did not expect the caregivers to understand this as an order to actually hasten their death.

\section{Implications for practice, theory, or policy}

- Patients expressing a desire to hasten death are in need of more information about the dying process.

- Medical staff providing this information can be helpful in managing the distressing anticipated images of agony.

\section{Introduction}

The diagnosis of a life-threatening disease such as cancer usually leads to the use of high-technology medicine, trying to cure the disease or at least slow down the progression as much as possible. In diseases where no cure is available yet (e.g. amyotrophic lateral sclerosis) or in those patients where treatment is not effective (e.g. recurrent cancer), the disease will progress to an advanced stage. For these patients, palliative care offers an option to control symptoms and preserve quality of life, making it possible for them to live their life as full as possible until the very end. Most patients with advanced progressive disease make good use of these options and try to come to terms with their reduced life expectancy.

However, some patients in this situation express a desire to hasten death. Different reasons have been suggested for this desire, ${ }^{1,2}$ and it seems clear that even though $10 \%$ of patients consider assisted suicide, only $1 \%$ of dying patients request it, and even where legal, only $0.1 \%$ receives and takes a lethal prescription. ${ }^{3}$ There seems to be a wide range with some patients who just seem to accept impending death and wait until death occurs naturally without hastening, up to others who ask for assisted suicide or euthanasia at the other end. Research on this subject is not always clear, as different terms (e.g. wish for hastened death, desire for death statements, and desire for early death) are used in the literature. ${ }^{1,4}$

Euthanasia (killing on request) is not allowed in German law, as detailed in Article 216 of the penal code. According to the same article assistance or encouragement of suicide is not punishable. The German legislation on assisted suicide is more liberal than that for Switzerland, where assisted suicide is a crime if and only if the motive is selfish according to Article 115 of the Swiss penal code. Physicians participating in physician-assisted suicide may be penalized by civil law. The German Medical Association has specified in their 2013 revision of the guideline regulations on specialty training that physicians are not allowed to perform euthanasia or assist with suicide. ${ }^{5}$ However, the 17 state chambers of physicians, with whom the legal authority rests, have transferred this paragraph in their regulations with different terminology, in some federal states changing or omitting this sentence. ${ }^{6}$

In the European neighborhood, Switzerland offers an option for assisted suicide, and several right-to-die organizations are active in Switzerland. Only Dignitas, EX International, and more recently LifeCircle also assist nonresidents with suicide. ${ }^{7}$

Hastening death is per definition not part of palliative care. Palliative care affirms life and regards dying as a normal process, it intends neither to hasten nor postpone death. ${ }^{8} 9$ Thus, a patient wishing to hasten death presents a huge challenge to palliative care professionals. This may be a burden to the relationship between care team, patient, and family and will require extensive communication with the patient and within the team and discussion of the underlying legal, philosophical, and ethical issues. ${ }^{6}$

In order to inform this discussion, we investigated the reasons and motivations of patients expressing a desire to hasten death in Germany. The focus was placed on the relationship between patient and team, asking specifically what patients expected from the health-care professionals when they expressed a desire to hasten death in a country with prohibitive legislation on euthanasia and physicianassisted suicide.

\section{Methods}

Potential participants were recruited from the departments of palliative medicine in three hospitals in Germany (University Hospital Aachen, University Hospital Cologne, and Malteser Hospital Bonn). Inpatients were recruited when they had made a statement or request to hasten death such as: "Please Doctor, give me some pills to stop waking up tomorrow!" or similar. Participants had to have an advanced incurable disease, aged over 18 years old, mentally not impaired, and were asked for informed consent. The study was approved by the Ethics Committees at all three institutions. 
The method for this qualitative study was a modified form of Grounded Theory. ${ }^{10,11}$ The main modification was the omission of theoretical sampling, as the number of patients with a wish for hastened death was small, and no larger pool of study candidates was available. The methodology and the modification have been described in detail elsewhere. ${ }^{12} \mathrm{~A}$ half-structured interview guideline was adopted, which allowed us to deepen the interview with specific topics. The interviews were held face to face and were audio taped. The aim was to explore the subjective world of experience of patients to better understand the wish to hasten death. Expectations toward the medical care providers were a second focus of the interview guideline. Sample size was determined by the concept of saturation. Interview partners were recruited until the point was reached at which information began to be repetitive with each additional subject. We stopped recruitment at 12 participants because of data saturation. The duration of the interviews depended on individual general condition and ability to cope.

Patients completed the Hospital Anxiety and Depression Scale (HADS) 13,14 and the Mini Mental Status Examination (MMSE) ${ }^{15,16}$ before the interview. Demographic and clinical data were collected with the Hospice and Palliative Care Evaluation (HOPE), ${ }^{17,18}$ which is part of the clinical standard documentation of the three participating services.

\section{Data analysis}

All interview transcriptions were analyzed by two female researchers (M.P. and S.S., both trained psychologists), both were supervised by a researcher group which included an ethicist, a palliative care physician, a psychologist, a sociologist, and a theologian. Both researchers were experienced in palliative care research using qualitative analysis and had not established a relationship with the study participants prior to the interview.

The constant comparison method was used to identify themes in the interview data. ${ }^{19}$ The software program MaxQDA was used for coding (grounded coding) and memorizing significant statements. The upcoming hypothesis and theoretical assumptions were differentiated, expanded, and revised with the data from subsequent interviews as long as no saturation of information was reached. As theoretical sampling was not possible, analysis of interviews from two outpatients, two patients with noncancer diseases, and two patients from the other study sites were performed last to check for confirmation of the information gained in the first set of interviews in these different settings as a substitute for theoretical sampling. ${ }^{12}$

A detailed report of the coding procedure as well as of methodological challenges and adaptations has been published previously. ${ }^{12}$ Selected data have also been published in a German review for continuous medical education. ${ }^{20}$

\section{Results}

\section{Participants}

Throughout the 26 months of the recruitment period (from January 2007 to March 2009) desire to hasten death statements were reported from 40 patients (from an estimated total of 1200 patients treated in this period in the three participating palliative care centers). Eight patients were excluded because of low cognitive function, low ability to cope with distress, or linguistic problems; 12 patients refused to participate; and another 8 patients were not included because of sudden death or organizational problems. ${ }^{12}$ Some patients did not meet the inclusion criteria, and others did not provide informed consent. Face-to-face interviews were performed in the palliative care units with 12 patients (10 inpatients and 2 outpatients). Participants included 5 women and 7 men with a mean age of 62 years (standard deviation $(\mathrm{SD})=12$ years, range $=38-78$ years). Two patients had chronic obstructive gastrointestinal syndrome with polyneuropathy and had been admitted to the palliative care unit for pain treatment. The other patients suffered from advanced cancer with a limited prognosis. Six patients were single, and six were married and had adult children. The mean duration of the interviews was $37 \mathrm{~min}(\mathrm{SD}=10 \mathrm{~min}$, range $=23$ $51 \mathrm{~min})$. The mean perception of distress during the interview was 2 on a numerical rating scale from $0=$ no distress to $10=$ maximum distress $($ range $=0-6)$. Even if patients reported a higher distress level, they emphasized that it was good to speak. As an example a patient reported, "yes, even though I have cried now, it was good to talk, yes at some moments it was releasing."

\section{Assessment scores}

Mean performance status (Eastern Cooperative Oncology Group (ECOG)) was 3, corresponding to being capable of only limited self-care and being confined to bed or chair for more than $50 \%$ of the waking hours. The mean result of the MMSE was $29(\mathrm{SD}=2$, range $=25-30)$. The HADS mean anxiety score was $6.5(\mathrm{SD}=5$, range $=1-15)$ and the depression score $10.5(\mathrm{SD}=6$, range $=2-19)$.

\section{Overview}

Patients provided a wide range of descriptions of life and illness. Strategies in coping, decision making, and the persistence of the desire to hasten death varied widely. Patients reported about their way of living, and provided information on coping strategies, decision making style in good and bad times, or managing responsibility. In several cases, although not in all, this provided explanations for the desire to hasten death as a consequence of their former lifestyle.

All patients knew that measures to hasten death such as physician-assisted suicide or euthanasia are not legal in Germany. One patient reported to be displeased about this. 
One patient went to Switzerland to receive assisted suicide assisted by the right-to-die organization Dignitas several weeks after his discharge from the palliative care unit. Nine patients discussed pros and cons and were able to accept the limitations of the legal situation in Germany at the same time.

The interviews provided a rich visual imagery of the experience of time and agony. Interestingly, all interviews were dominated by the use of expressive language and of superlatives. Nearly, all patients described very vivid pictures about anticipated situations which they tried to avoid. Patients told us that this was their first opportunity to speak about their thoughts.

Patients reported that they wanted their thoughts to be explored with respect to their feelings in the context of death and dying. The most important aspect was that physicians were asked to give affection, trust, and communicate about patients' ideas and perceptions in an atmosphere of respect.

Thematic analysis (Figure 1) revealed three main motivational themes (self-determination, agony and time) that led to the emerging core category of time. Attitudes toward death and secureness related to end of life were other main themes that seemed less relevant for the core category and the generation of theory. Expectations toward medical care providers emerged as a separate theme from the analysis, as this was a separate focus of the interview guideline.

\section{Self-determination}

The main codes related to the theme self-determination were responsibility, changes in life, and support by relatives or resources. The theme self-determination seemed to be essentially characterized by the wish for control over the situation in which patients found themselves. The progress of the disease with death at the end was described as leading to a forced choice. Patients felt that a decision about the continuation or ending of their life would be too hard for others (relatives and caregivers) and that this decision would have to be done by themselves. Leaving their things in good order when they have died was important. Time was described as a chance for the patient to exclude some options how things could go on, this is meant as a responsible and preventive act:

They do not feel the agonies, but I do. Now it has come so far, that I am only lying here. You think. You think and think and you bring many things to mind and passing in revue I have come to a decision, yes, well ... I am lying waiting for death. (P11)

The meaning of control is directly related to order as an antagonism to illness (=disorder). Illness is felt as heteronomy ("Fremdbestimmung") and chaos by unintended changes in life, changes of something that had been important for their way of living before the disease had struck.
They felt the loss of typical interests or activities or the need to submit long- and short-term personal goals to the disease:

Activities with friends not possible - and indeed activities outside or with the family, I would say, meanwhile completely passed away. (P7)

Patients addressed problems with the support from family members. They emphasized that the family members were providing care over a long period of time. This was described, on one hand, as a source for thankfulness; on the other hand, this aspect seemed to be a source of a guilty conscience and loss of self-esteem:

This is a bit of a vicious circle (Teufelskreis). When my wife sees that I am doing badly then that makes her suffer. Then I see that my wife is suffering severely and then I-because I know that basically it is my fault. If you can talk about a fault. (P2)

Some wanted to avoid that their loved ones are going to get physically or psychologically ill through caring for them over time. Others feared to lose their self-esteem while they perceive the interaction with others as superimposed by images of not being the same person anymore:

I am not used to somebody helping me. This I feel is tantalizing, this is no life. (P8)

\section{Agony}

The most central aspect within the theme "agony" was the anticipation that at some point in time, one could not express his needs, wishes, or problems anymore because of apathy, strong weakness, or cognitive impairment:

... but really yes, to see a man, who only exists of pain, who maybe is cognitively impaired and isn't able to participate actively in life, this man, who only lies in bed, not noticing his surroundings ... (P2)

Patients reported about bad and painful experiences throughout their illness trajectory, and as vivid as if it had happened, they anticipated a future state of being in agony and harm, described by a rich imagery and using extreme words (e.g. with superlatives such as perishing in the most horrendous condition). They reported concerns about loss of physical functions as related to prior activities. This was linked directly to self-determination and changes in life. They feared that they would experience starving and physical infirmity. Patients stated that their body had been telling them to hasten death. The ongoing as well as the anticipated loss of body functions in the future led them to the desire to hasten death. In this context, patients spoke about a prolonged dying process, merely vegetate, or lie idle.

Patients reported that they felt like dying now and that their bodies will rot away. Dying meant for the patients a 


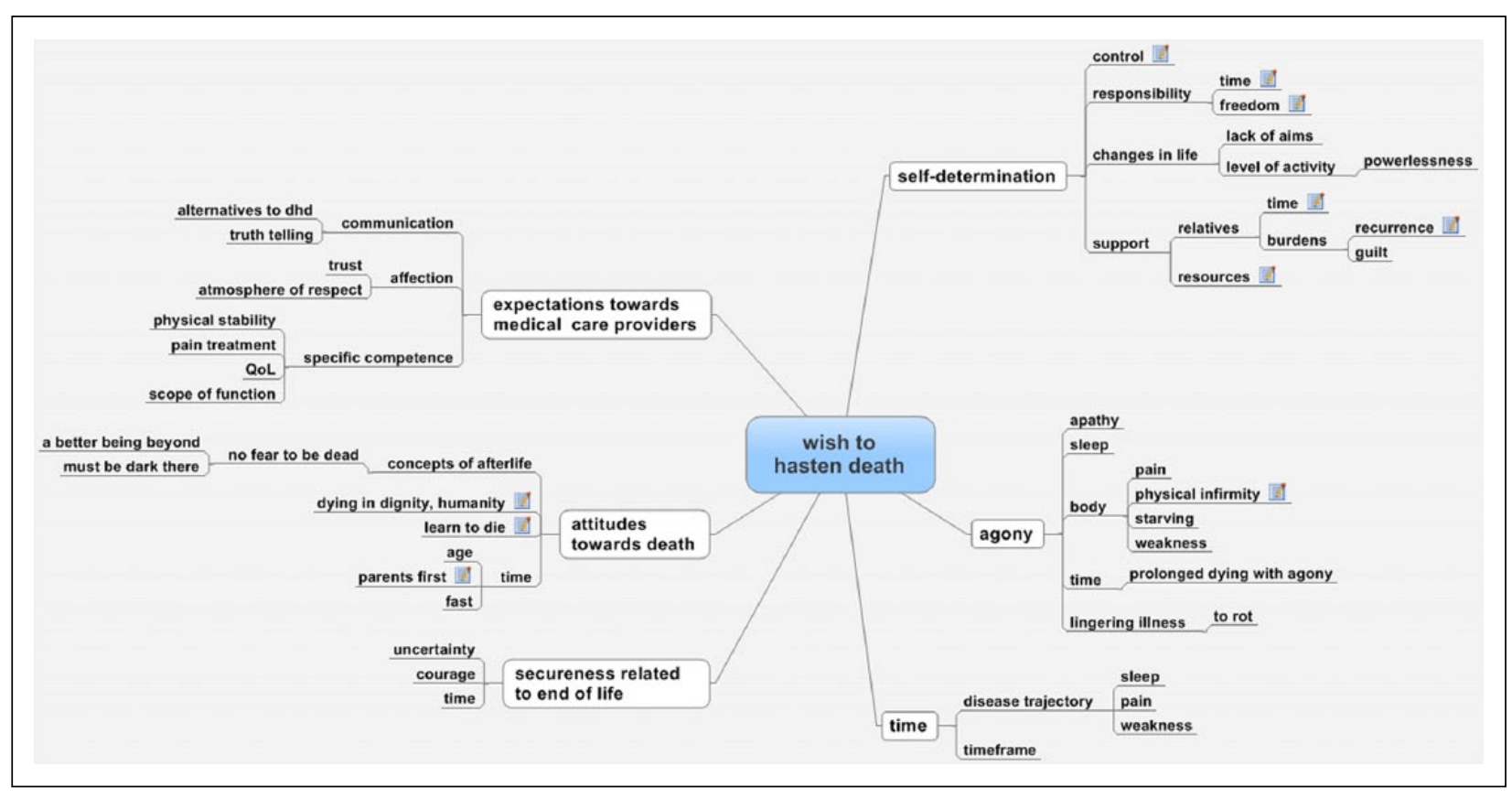

Figure I. Overview on themes from qualitative analysis of interviews with 12 patients.

process with extended borders. Some described their dying process as enduring since months:

I am not afraid about death. I am only afraid of an agonizing death. Taking too much time ... (P9)

Others anticipated that it would take too much time to die, and therefore, it was expected to be horrible. All in all, they described images of a lingering illness and significant weakness, expecting an awful future ending with death.

\section{Time}

The perception of time was described as a struggle between time periods characterized as too fast or too slow and as too short or too long within the disease trajectory. In terms of the progression of the disease, this was expressed as all of a sudden everything was over, and there were long periods of suffering. Patients thought about their future as a long way in agony:

I knew that I have half a year at most, a year, not more. (P9)

All emerged themes were interwoven with expressions related to time, suggesting time as the emerging core category of the study. The aspect of self-determination was superimposed and influenced by the sense of time, which was, on one hand, passing too slowly, so that patients felt every minute, and it gave them much time to consider the situation, and on the other hand, time was restricted too much by the prognosis, which made them consider life as a time line with boundaries. Patients even reported the feeling that time was too long for their relatives:
And I would not want to go through this as a daughter (of a patient). Sit down every day and watch how somebody is running towards death, lying there and finally waiting to be released. (P7)

The aspect of agony was also interlaced with expressions related to time such as waiting and waiting, too often, extended, prolonged, so long, on and on, it should be over, limited, until the last moment, and from one second to another. One patient reported that he felt like being dead already. A physician had told him that he should have been dead for some months or years by now.

Looking back brought memories of special problems with symptoms or treatments as sequences in life. Patients reported about times of really bad pain or a specific point in time such as since then I couldn't walk anymore or from that point on I knew life time was limited.

\section{Secureness related to end of life}

The theme secureness related to end of life was sometimes described as a feeling of uncertainty, as not being able to decide or plan for one's own life:

Yes, and that is this uncertainty. You can plan nothing and, let me say, only on short notice, for very short distances. (P9)

Others described a feeling of safety while being treated in the specialist inpatient unit. Safety was also an issue when patients wanted to have the means for suicide available but for the future, not for right now. This would provide a sense of security when the disease burden is high: 
If I would have such a pill in some drawer or the other, and I could take it on my own when I would have the feeling that nothing goes any more, then I would feel considerably better. (P9)

\section{Attitudes toward death}

Patients reported different concepts of an afterlife, either affirmative (I do believe that there is something to come after death) or negative (life after death, I do not really believe that). Patients often were seeking for spiritual beliefs:

Perhaps this can help me to develop a phantasy for myself, what perhapy may be thereafter. (P10)

Patients not only thought about death and afterlife but also about dying, for example, describing that they wanted to die with dignity.

\section{Expectations toward medical care providers}

Patients explained that the intention to address their desire to hasten death to medical staff as their palliative care provider was associated with the need for security and the perception of competence in symptom control.

Patients reported feeling a lack of communication on their desire to hasten death, with the health professionals as well as with family members and friends. Expressing the desire to hasten death corresponded with their need to be apprehended and accepted. Patients' expectations toward the caregivers were to explore their ideas and perceptions and respect their experiences. They did not want staff to consider this ideation as a psychiatric problem but rather as part of a normal process in dying. The main helpful intervention for patients was for medical staff to listen: I just wanted to get rid of it. Patients did not expect medical staff to execute their request and end their lives:

And let us see, depending on whom you tell it some new alternatives will be presented. (P7)

Some patients discussed the risks of suicide using prescribed medicines. They were able to discuss the disadvantages if substances or dosages were not judged accurately enough. They identified a need for expertise in this field to get a safe and controlled death without suffering:

This is such an uncertain thing. And with such a toxic cocktail you are on the safe side, I would say. Then you know exactly, aha there it is, you can take it or have it given to you, you can decide all this in advance, in written and then the ordeal is shortened. (P7)

At the same time, they discussed the risks for the caregiver-patient relationship and a potential loss of trust was admitted. As a consequence, one patient told us that he and his family members had to learn to die and that they needed support by talking about the individual dying process and how to say goodbye with medical staff.

\section{Discussion}

In this study, patients were asked about their biographies and their own understanding of their wish to hasten death, resulting in rich information about patients' life and disease trajectories and in some cases providing explanations for the desire to hasten death as a consequence of their former lifestyle.

The main motivations for the desire to hasten death were grouped around the themes self-determination, agony, time, secureness, and attitudes toward death. In the evaluation of interviews with palliative care patients who had made a statement or request to hasten death, several hypotheses emerged, related to the core category of time:

1. Patients are involved in an active process of constructing the course of disease. The sense of self is modified by this process;

2. Patients try to balance life time and anticipated agony. In this balancing act, the perception of time is distressing;

3. Anticipated images of agony and suffering in the dying process occur frequently. Patients experience them as intrusive;

4. The desire to hasten death is an extreme coping strategy for intrusive emotional and cognitive experiences;

5. Patients expressing a desire to hasten death are in need of more information about the dying process. Medical staff providing this information can be supportive in managing the distressing anticipated images of agony;

6. Patients do not perceive their desire to hasten death and the provision of palliative care as mutually exclusive. Caregivers should be able to explore the ideation to hasten death and respect the patient's experience of the disease.

The main motivations found in this study correspond well with other studies on the subject. A systematic review on the reasons for desire to die requests found 35 research studies that met the search terms. ${ }^{2}$ Being a burden to others, loss of autonomy, physical symptoms, depression and hopelessness, existential concerns, and fear of the future were the most commonly named reasons. However, most of these studies reported on patients' opinions about why they may have a desire for hastened death in the future or about family members or health-care professionals' opinions of patients' reasons. Only eight studies, none of them from Europe, provided reasons from patients who had 
made a desire to die statement. The main reasons in these studies were being a burden to others (included in the theme of self-determination in this study), physical symptoms (included in the theme agony), and fear of the future (included in the theme time).

Time emerged as the core category in the evaluation of our study. All themes were interwoven with expressions related to time. Our results demonstrated that anticipated agony, and not the suffering patients experience right now, seemed more prominent in the generation of the desire to die. This fits well with the review of Hudson et al. ${ }^{2}$ reporting that psychosocial and existential factors were common than those directly related to physical symptoms such as pain. Concerns about future distress were specifically listed as major reasons for desiring death in a study in Japanese $^{21}$ and US ${ }^{22}$ cancer patients. In a nationwide survey in France, palliative care teams also reported physical symptoms such as pain only in a small minority of cases as the reason for the desire to die but much more often difficulties with feeding, movement, excretion, or other signs of deteriorating body functions. ${ }^{23}$

A more recent qualitative review on the meaning and motivation for the wish to hasten death in patients with chronic illness or advanced disease interpreted results from seven studies and found six emerging themes: response to physical, psychological, spiritual suffering; loss of self; fear of dying; the desire to live but not in this way; a way of ending suffering; or control over one's life. ${ }^{1}$ Two of the studies included in this review had used Grounded Theory as methodology. ${ }^{24,25}$ The authors developed an explanatory model which showed the wish to hasten death to be a reactive phenomenon in response to multidimensional suffering rather than any single aspect of suffering. The main factors in the model were total suffering, loss of self, and fear, which again correspond well to the themes of self-determination, agony, and time we found in our evaluation.

Both reviews included studies from the United States, Canada, Australia, and Asia but no studies from Europe. Only recently has a Swiss report described the intentions and motivations of a wish to die in patients with terminal cancer. ${ }^{26,27}$ However, the authors included patients with and without a wish to die, and there is no information how many patients of the study sample did explicitly request hastened death. The authors describe a wide range of nine different intentions (ranging from the will to live to acting toward dying) and a similar range of motivations, including different reasons, meanings, and functions of the wish to die, including being an example to other on how to die, spare others the burden of oneself, re-establishing agency, or manipulation. The main themes self-determination, agony, and time are represented by analogous meanings in the Swiss study. Differences in the legislation between Switzerland and Germany did not seem to make a fundamental difference on the motivations of the patients. Only for one of the nine meanings (preserve self-determination), it is mentioned explicitly that all four patients in this group were members of a right-to-die organization. In our study, only one patient was a member of such an organization.

Our report on motivations of patients with the desire to hasten death confirms the international findings, including the Swiss study. In addition, we present information on the patient's expectations toward the medical care providers from a European setting.

In Germany, euthanasia and physician-assisted suicide are penalized. This does not mean that these practices are nonexistent, as there may be a significant number of unreported cases. An anonymous survey comparing six European countries found that physicians reported intentionally hastening death on the explicit request of a patient less frequent in countries where this was not legal (Austria, Denmark, Sweden, and Italy), but the prevalence in these countries still ranged from $1 \%$ to $14 \%$ of the participating physicians.

However, for countries with no legal options to hasten death, information about patients' expectations toward medical staff when they are expressing a desire to die is missing. Our study clarified that patients needed an offer to talk about their desire to hasten death. Patients seemed to require more attention from medical staff for their desire to hasten death. They did not expect team members to understand their statement as an order to actually hasten their death. Only one patient selected the option to travel to Switzerland and received assisted suicide several weeks after his discharge from the palliative care unit.

We were deeply impressed that all patients claimed that both health and family caregivers tended to ignore or deny their desire to die. This leads to the assumption that it would be very helpful for patients if caregivers would be able to listen to and respect wishes related to hastened death. Without trying to change the desire to hasten death, health professionals should be supportive in managing the distressing anticipated images of agony. The ideation of the desire to die could be understood as an individual measure against psychological distress.

Schwarz ${ }^{28}$ has criticized the mechanical repetition of the hospice movement's credo that palliative care neither hastens nor postpones death, which might leave patients feeling alone and misunderstood. She provides an example of a hospice nurse answering "I can't help you with that" and pushing away from the table in response to a desire to hasten death statement. She postulates that medical staff caring for terminally ill patients should encourage patients who ask about all legally and ethically sound palliative options, including those that might hasten death, such as forgoing life-prolonging interventions or refusing food and fluids. However, such advice is often hampered by fear of subsequent allegations of euthanasia or physicianassisted suicide..$^{29}$

The need for careful and ongoing communication with health professionals who are skilled in therapeutic communication is emphasized in the recommendations of Hudson et al. ${ }^{30} \mathrm{~A}$ two-phase approach is recommended, 
with health professionals taking time to explore the background to the desire to die statement first (phase 1) before offering strategies or providing detailed information (phase 2). This approach should convey empathy for the patient's distress. Using this approach, health professionals may find it easier to determine whether a desire to die statement is about a request for hastened death, a sign of psychosocial distress, or merely a passing comment that is not intended to be heard literally as a death wish. This is confirmed by the model developed in the meta-ethnography by Monforte-Royo et al., ${ }^{1}$ which found the wish to hasten death to have different meanings, which do not necessarily imply a genuine wish to hasten one's death.

Hudson et al. also clarify that where relevant, referral to health professionals who specialize in management of psychosocial distress is necessary. This is even more important as psychiatric disorders should not be overlooked in this patient group. Cancer patients with a high wish to hasten death were significantly more likely to have a current (or past) major depressive episode compared to patients with no such wish. ${ }^{31}$ However, the desire to die is not simply a feature of depression in terminally ill patients. In a study on motor neuron disease, patients who hastened dying reported a reduction in suffering and increased perception of control over the disease in the final weeks of life. ${ }^{32}$

Our study results also suggest that patients need more information on the dying process, as the patients anticipated dying in agony, with little information on palliative care options, but also much uncertainty about the end of life at the same time. It would be important to understand whether the provision of knowledge about the dying process could cause anxiety and despair in these vulnerable patients or rather would provide a sense of security and trust. This kind of psychosocial symptom management could stabilize patients in the present instead of letting them linger with anticipated agony in the future. Hudson et al..$^{30}$ emphasize that the provision of comprehensive information about what to expect in the future promotes psychological well-being, but no research on this topic is available.

The desire to hasten death may be used by patients to maintain control against the anticipated agony. Patients did not feel that their desire to hasten death and the provision of palliative care were mutually exclusive. They expected medical caregivers to listen to and respect their experiences. Research is needed on interventions to improve communication, diminish psychological distress, and foster the sense of self-determination in these patients.

\section{Acknowledgements}

We thank the participating patients, their relatives, and all caregivers from the palliative care units in Aachen, Bonn, and Cologne for supporting the study and dedicating time and energy. We thank all associated researchers for their helpful support, review, and comments to this article. Research material related to this article can be accessed via the corresponding author.

\section{Declaration of conflicting interests}

The authors declare that there is no conflict of interests.

\section{Funding}

This study was supported by a grant of the Deutsche Forschungsgemeinschaft (DFG, 350253).

\section{References}

1. Monforte-Royo C, Villavicencio-Chavez C, Tomas-Sabado $\mathrm{J}$, et al. What lies behind the wish to hasten death? A systematic review and meta-ethnography from the perspective of patients. PLos One 2012; 7: e37117.

2. Hudson PL, Kristjanson LJ, Ashby M, et al. Desire for hastened death in patients with advanced disease and the evidence base of clinical guidelines: a systematic review. Palliat Med 2006; 20: 693-701.

3. Bascom PB and Tolle SW. Responding to requests for physician-assisted suicide: "These are uncharted waters for both of us.” JAMA 2002; 288: 91-98.

4. Monforte-Royo C, Villavicencio-Chavez C, Tomas-Sabado $\mathrm{J}$, et al. The wish to hasten death: a review of clinical studies. Psychooncology 2011; 20: 795-804.

5. Bundesärztekammer. (Muster-)Berufsordnung für die in Deutschland tätigen Ärztinnen und Ärzte (Stand 2011). Berlin: Bundesärztekammer, 2011, http://www.bundesaerztekammer. de/page.asp?his=1.100.1143 (accessed 12 March 2014).

6. Nauck F, Ostgathe C and Radbruch L. Ärztlich assistierter Suizid: Hilfe beim Sterben-keine Hilfe zum Sterben. Deutsches Ärztebl 2014; 111: A67-A71.

7. Schmidlin E, Elger BS and McLennan S. Assisted suicide in Switzerland: where do we come from and where are we going? Eur J Palliat Care 2014; 21: 61-65.

8. Sepulveda C, Marlin A, Yoshida T, et al. Palliative Care: the World Health Organization's global perspective. J Pain Symptom Manage 2002; 24: 91-96.

9. Radbruch L, Payne S, Bercovitch M, et al. White paper on standards and norms for hospice and palliative care in Europe part 1-recommendations from the European Association for Palliative Care. Eur J Palliat Care 2009; 16: 278-289.

10. Glaser AW, Abdul Rashid NF, U CL, et al. School behaviour and health status after central nervous system tumours in childhood. Br J Cancer 1997; 76: 643-650.

11. Glaser BG and Strauss A. The discovery of grounded theory: strategies for qualitative research. Chicago, IL: Aldine, 1967.

12. Stiel S, Pestinger M, Moser A, et al. The use of Grounded theory in palliative care: methodological challenges and strategies. J Palliat Med 2010; 13: 997-1003.

13. Zigmond AS and Snaith RP. The hospital anxiety and depression scale. Acta Psychiatr Scand 1983; 67: 361-370.

14. Herrmann C. International experiences with the Hospital Anxiety and Depression Scale - a review of validation data and clinical results. J Psychosom Res 1997; 42: 17-41.

15. Folstein MF, Folstein SE and McHugh PR. Mini-MentalState: a practical method for grading the cognitive state 
of patients for the clinician. $J$ Psychiatr Res 1975; 12: 189-198.

16. Folstein MF, Folstein SE, McHugh PR, et al. Mini-MentalStatus-Test (German version). Weinheim: Beltz Test GmbH, 1990.

17. Stiel S, Pollok A, Elsner F, et al. Validation of the symptom and problem checklist of the German Hospice and Palliative Care Evaluation (HOPE). J Pain Symptom Manage 2012; 43: 593-605.

18. Lindena G, Nauck F, Bausewein C, et al. Qualitatssicherung in der Palliativmedizin - Ergebnisse der Kerndokumentation 1999-2002. Z Arztl Fortbild Qualitatssich 2005; 99: $555-565$

19. Lacey A and Luff D. Qualitative research analysis. Sheffield: NIHR RDS for the East Midlands / Yorkshire \& the Humber, 2009, http://www.rds-eastmidlands.nihr.ac.uk/ resources/cat_view/13-resource-packs.html (accessed 8 November 2012).

20. Stiel S, Elsner F, Pestinger M, et al. Wunsch nach vorzeitigem Lebensende: Was steht dahinter? Schmerz 2010; 24: 177-189.

21. Morita T, Sakaguchi Y, Hirai K, et al. Desire for death and requests to hasten death of Japanese terminally ill cancer patients receiving specialized inpatient palliative care. $J$ Pain Symptom Manage 2004; 27: 44-52.

22. Pearlman RA, Hsu C, Starks H, et al. Motivations for physician-assisted suicide. J Gen Intern Med 2005; 20: 234-239.

23. Ferrand E, Dreyfus JF, Chastrusse M, et al. Evolution of requests to hasten death among patients managed by palliative care teams in France: a multicentre cross-sectional survey (DemandE). Eur J Cancer 2012; 48: 368-376.
24. Lavery JV, Boyle J, Dickens BM, et al. Origins of the desire for euthanasia and assisted suicide in people with HIV-1 or AIDS: a qualitative study. Lancet 2001; 358: 362-367.

25. Nissim R, Gagliese L and Rodin G. The desire for hastened death in individuals with advanced cancer: a longitudinal qualitative study. Soc Sci Med 2009; 69: 165-171.

26. Ohnsorge K, Gudat $\mathrm{H}$ and Rehmann-Sutter C. What a wish to die can mean: reasons, meanings and functions of wishes to die, reported from 30 qualitative case studies of terminally ill cancer patients in palliative care. BMC Palliat Care 2014; 13: 38 .

27. Ohnsorge K, Gudat H and Rehmann-Sutter C. Intentions in wishes to die: analysis and a typology - a report of 30 qualitative case studies of terminally ill cancer patients in palliative care. Psychooncology 2014; 23: 1021-1026.

28. Schwarz J. 'I can't help you with that': when terminally ill patients wish to hasten their dying, nurses can-and should-help. Am J Nurs 2008; 108: 11.

29. Szalados JE. Discontinuation of mechanical ventilation at end-of-life: the ethical and legal boundaries of physician conduct in termination of life support. Crit Care Clin 2007; 23: 317-337, xi.

30. Hudson PL, Schofield P, Kelly B, et al. Responding to desire to die statements from patients with advanced disease: recommendations for health professionals. Palliat Med 2006; 20: 703-710.

31. Kelly BJ, Pelusi D, Burnett PC, et al. The prevalence of psychiatric disorder and the wish to hasten death among terminally ill cancer patients. Palliat Support Care 2004; 2: 163-169.

32. Albert SM, Rabkin JG, Del Bene ML, et al. Wish to die in end-stage ALS. Neurology 2005; 65: 68-74. $\Psi$ 